\title{
The Evolution of the National Geodetic Survey's Continuously Operating Reference Station Network and Online Positioning User Service
}

\author{
William Stone \\ National Oceanic and Atmospheric Administration - National Geodetic Survey \\ MSC01-1110 \\ 1 University of New Mexico \\ Albuquerque, NM 87131 USA
}

\begin{abstract}
The United States Department of Commerce's National Geodetic Survey (NGS) has developed a nationwide network of continuously operating reference stations (CORS) designed to support the broad spectrum of post-processed, relative Global Positioning System (GPS) techniques and applications. This network now comprises more than 900 permanent, geodetic-quality GPS receivers, in various installation configurations. The network was established through an exemplary cooperative effort, involving nearly 200 organizations from various government levels, academia, and the private sector. CORS GPS observational data are freely provided to the user community via the Internet and are capable of supporting high-accuracy positioning requirements. In addition to enhancing geospatial positioning, applications of CORS data include the following: a critical role in defining the nation's geodetic reference system; the ability to characterize the free electron content of the ionosphere; and an important source of precipitable water vapor input to meteorological forecasts. Associated with the CORS network, the Online Positioning User Service (OPUS) is a free, automated Web-based utility that processes submitted GPS observation data with respect to the CORS network, providing corresponding positional coordinates, via email, usually in minutes. This powerful tool has proven to be very popular with the geospatial community and is changing the way NGS is able to provide access to the National Spatial Reference System.
\end{abstract}

\section{INTRODUCTION}

The National Geodetic Survey (NGS), an office of the United States Department of Commerce's National Oceanic and Atmospheric Administration (NOAA), defines and manages the National Spatial Reference System (NSRS) - the nation's geographic coordinate system. Through its definition of latitude, longitude, and height (and other geodetic parameters), the NSRS provides a multi-purpose, coordinatebased foundation that supports the broad spectrum of today's three-dimensional positioning applications.

February 10, 2007, marks the two-hundredth anniversary of the establishment of NGS (previously called the Survey of the Coast and, later, the Coast and Geodetic Survey), which came into being as a direct result of the success of Lewis and Clark's Corps of Discovery expedition through western America. During most of the two-century evolution of the NSRS, access to the system has been provided by a network of passive, monumented ground control points. These geodetic control stations have historically had their horizontal and/or height coordinates determined by conventional optical and mechanical surveying techniques and geodetic computations. Although ground control points are still in use today, the realization of, and access to, the NSRS has been dramatically modernized by surveying applications of the Global Positioning System (GPS).

Since the mid-1980s, the use of GPS for the establishment of precise geodetic control has grown steadily. Today, conventional horizontal-positioning techniques and equipment are rarely used for such work. Since 1994, NGS has managed the development and operation of a multiorganization network of permanently installed, survey-grade GPS reference stations, known as continuously operating reference stations (CORS). The CORS network supports three-dimensional, centimeter-accuracy GPS positioning and other GPS-based applications in the United States and its territories, as well as in several collaborating foreign countries. Furthermore, this system represents a modernized means for surveyors, geographic information system (GIS) professionals, researchers, and others to actively access the NSRS through computed station coordinates and velocities, and GPS observational data, available free of charge, for postprocessed GPS applications.

In 2001, NGS furthered the functionality and value of the CORS network by developing a related Web-based utility, the Online Positioning User Service (OPUS), to provide an automated method of determining user positions relative to the CORS network. OPUS provides fast, accurate, reliable, and consistent access to the NSRS via a simple Web interface. OPUS requires a user to simply collect dual-frequency GPS data at a location whose coordinates are desired, then upload the observations to the utility. Within minutes, the user will receive an email containing the OPUS-derived coordinates for the submitted GPS data file.

Reference [1] discusses the status of CORS and OPUS in 2002 and helps provide a perspective to the evolution of these programs in the past few years. As the basic technology of GPS has improved with time, CORS and OPUS have both grown and matured as well. 


\section{CONTINUOUSLY OPERATING REFERENCE STATION NETWORK}

\section{A. Network Development}

The CORS network began with NGS' early 1994 installation of a permanent GPS station on the Gaithersburg, MD, campus of today's National Institute of Standards and Technology - an appropriate site for the nascence of what has evolved into the de facto national positioning infrastructure standard. By the end of 1994, the CORS network had grown modestly to a total of five stations, located around the country. The network's growth has accelerated substantially with time and in 2005 more than 200 additional stations were incorporated into the system, edging the total toward the 1,000-site milestone, which will soon be achieved. Fig. 1 is a map of CORS locations, showing the network status in April, 2006.

Personnel involved in the development of the CORS network realized, from the program's outset, that with its limited, small-agency resources, NGS would be unable to single-handedly build the envisioned network. Consequently, the program continues to rely on successful partnerships with other organizations to help realize the goal of building a national, multiple-use, and robust reference station network. NGS presently owns and operates less than three percent of the sites. The majority of the stations were installed by various local, state, and federal government agencies, academic institutions, and the private sector. CORScollaborating organizations, now numbering close to 200, establish permanent reference stations to support their own varied requirements and applications - surveying and mapping, real-time navigation, geophysical research, and atmospheric modeling, to name some of the more prevalent uses. By participating in the CORS program, these site collaborators can realize a number of added direct benefits to their GPS programs - including data quality control, distribution, and archive functions.

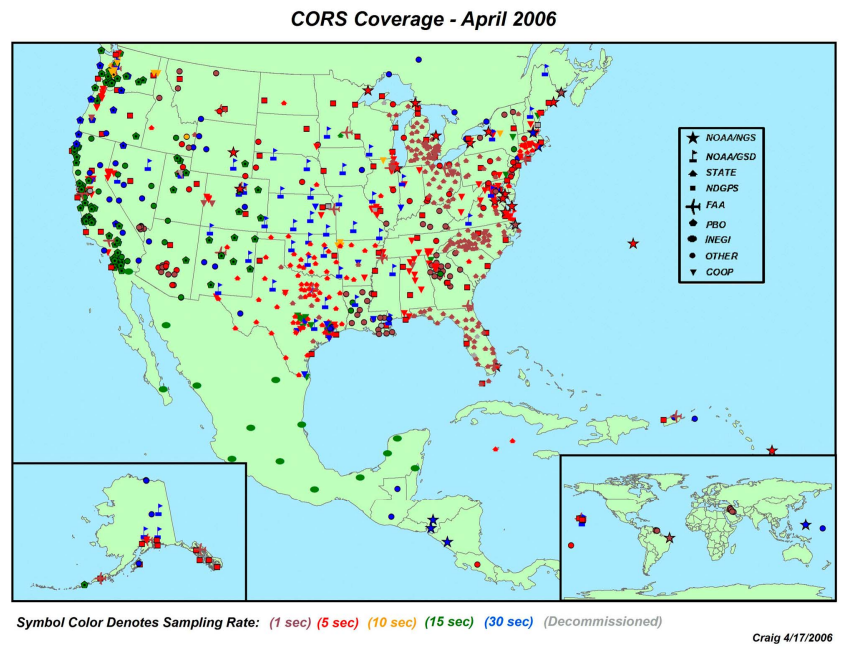

Fig. 1. Map of CORS locations (April, 2006). Station symbols and colors indicate the establishing organization and data sampling rate, as noted. Legend acronyms are defined in the text (except COOP (Cooperative CORS)).
There are far too many CORS network collaborators to discuss them and their sub-networks individually; however a few of the participating programs and organizations will be mentioned, due to the scale of contributions they are making to the program.

First, the United States National Science Foundation has funded an ambitious, multi-year research effort - EarthScope - with goals aimed at furthering our understanding of the physical processes involved in the structure and evolution of North America. One component of EarthScope, the Plate Boundary Observatory (PBO), is designed to be a geodetic observatory focused on studying the inter-tectonic plate, deformation-induced strain field in the western United States. A crucial observational tool of PBO is a network of continuous GPS sites that will eventually grow to about 900 installations. Many of these PBO sites have already been incorporated into the CORS network, with more to follow, as they are installed.

Several other institutions in the geophysical research community have established regional GPS networks to monitor crustal deformation-induced motions - efforts that are similar to PBO's undertaking, but on more localized scales.

Second, NOAA's Global Systems Division (GSD) has installed dozens of CORS sites. Many of these are collocated with meteorological monitoring instrumentation, and they participate in a nationwide network of nearly 400 sites, used to determine atmospheric moisture content.

Third, the United States Coast Guard and Department of Transportation are working cooperatively to expand the Coast Guard's existing real-time GPS maritime navigational beacon system to provide nationwide differential signal coverage, in support of safe and efficient terrestrial navigation. Stations in this Nationwide Differential GPS (NDGPS) network, now numbering nearly 100 , will serve double-duty by also participating in the CORS network.

Fourth, the Federal Aviation Administration's (FAA) Wide Area Augmentation System (WAAS) is a satellite-based, realtime correction system, designed for aircraft navigation, but is frequently used in terrestrial-based applications as well. All of the ground reference stations of the WAAS infrastructure are also feeding data into the CORS system.

Fifth, the National Aeronautics and Space Administration (NASA) has been establishing permanent GPS installations for many years. Many of these sites, used for various space applications and research, have been incorporated into the CORS network.

Finally, many state governments, including Florida, Michigan, North Carolina, Ohio, Oklahoma, and Texas (each currently has about 20 or more sites), have established statewide or large-region GPS networks to support their own positioning applications, either post-processed or real-time. These statewide efforts are generally the work of the state's department of transportation (except for the North Carolina Geodetic Survey) and many also contribute to the CORS network. Several other states are currently in the planning stages of developing their own similar statewide GPS-based infrastructure. 
In addition to providing coverage for the nation, the CORS network has spread to several foreign countries, furthering the unique collaborative nature of the program. Both Mexico's Instituto Nacional de Estadistica, Geografia e Informatica (INEGI) and Canada's Natural Resources Canada (NRCan) have established stations that have been incorporated into the CORS network. These sites contribute greatly to large-area, CORS-based modeling applications in the United States that might otherwise suffer from adverse "boundary condition" weakness, caused by proximity to an edge of the coverage area. Nearly a dozen nations in the Caribbean Sea region, Central America, and South America also participate in the program. NGS worked closely with the United States Army to establish a six-station network in Iraq to support that nation's ongoing reconstruction process.

\section{B. Site Configurations}

In order to provide the requisite high level of CORS data quality and consistency needed to support a broad spectrum of user applications, including the vital role that the network plays in the realization of the NSRS, NGS has rigorous site standards and installation criteria that must be met by potential collaborators, before a station can be included in the network. Reference [2] provides the latest set of site requirements and recommendations for the establishment and operation of a CORS site. These specifications were developed with the goals of providing an optimal GPS signal collection environment, the highest possible quality in computed station coordinates and velocities, and the required site documentation for users. As the document explains, site selection decisions are made on an individual basis and consider not only site quality details, but overall network issues, including regional station coverage, site infrastructure robustness, and other requirements. An NGS site selection team meets regularly to review and consider potential additions to the CORS network, submitted by interested organizations.

Because CORS sites are installed and operated by individual collaborators, they come in many different configurations, each tailored to meet the native application requirements established by the site owner. The fundamental site and installation criteria that must be satisfied to participate in the network can be summarized as follows (see the guidelines document for more details and the complete list):

- stable, well-anchored monument and antenna mount

- minimal visibility obstructions (none $>10$ deg elevation)

- benign signal environment (no interference or multipath)

- L1/L2 GPS receiver tracking > 10 satellites

- L1/L2 NGS-calibrated GPS antenna

- L1 C/A- or P-code pseudorange observations

- L1 and L2 full wavelength carrier phase observations

- $\leq 30$ second epoch interval

- data logging hourly, daily, or in real-time stream

- uninterruptible power supply capability

The CORS network is divided into two sub-networks National CORS, which is by far the larger component, and Cooperative CORS. Each CORS is assigned to one of these network categories depending on how a user accesses the site's data, as described in the following section. Other than this distinction, there are few substantive differences between the two CORS varieties, and a detailed comparison is included in the guidelines document.

Fig. 2 is an example of a CORS installation.

\section{Data}

Researchers in various disciplines have developed many creative and significant applications of the CORS network during the dozen years the system has been evolving. However, the network's fundamental purpose, from the outset, has been to provide geospatial users with GPS observation data that can be incorporated into post-processed user positioning applications, primarily in surveying, mapping, and related disciplines. The CORS data augment users' GPS observation data by providing a direct connection to the highest accuracy realization of the NSRS and a corresponding direct link to precisely determined station coordinates.

Users access the GPS observation data collected at National CORS directly from NGS, via anonymous ftp (ftp://www.ngs. noaa.gov/cors/) or the World Wide Web (http://www.ngs. noaa.gov/CORS/). Whereas access to the Cooperative CORS network data is provided directly by the establishing organization, via a Web page (http://www.ngs.noaa.gov/ CORS/Coop/), that provides links to Web sites of all the cooperating entities. A number of CORS installations fall into both categories and are referred to as "Combo CORS," to reflect the fact that data can be accessed from either NGS or the host organization.

CORS observation data files consist of GPS code and carrier phase observations, which are provided in the universally recognized Receiver Independent Exchange (RINEX) format. Users retrieving data via the World Wide Web interface have two different options for requesting data -

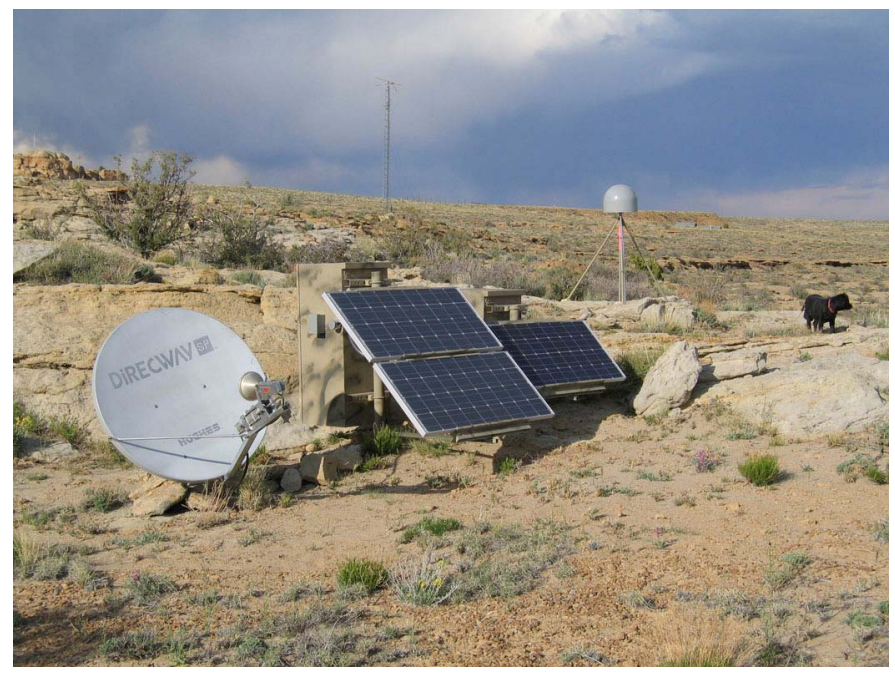

Fig. 2. CORS site P028, located in Chaco Canyon, NM, and established/owned by the Plate Boundary Observatory. Pictured are: satellite dish for data communications, solar panels, equipment housing boxes, and GPS antenna attached to anchored mount. 
the user-friendly CORS (UFCORS) and the standard download interfaces. UFCORS provides the user with the ability to: request a data file covering a desired time period (including, for instance, a time block that spans multiple calendar days); have it populated at a specified epoch interval; have the observations packaged along with various site metadata and ancillary GPS information; and to have all of this information file-compressed, as desired. Although the same information, including the ancillary data, can be accessed via the standard download interface, this approach can involve multiple manual operations on the part of the user. UFCORS is extremely easy to use and has proven to be popular. UFCORS data requests currently average approximately 40,000-60,000 per month, with typical total data download volume of 30-50 gigabytes. This traffic represents an approximately ten-fold increase, compared with typical monthly activity in 2000 .

Anonymous $\mathrm{ftp}$ is the most popular method, in terms of data volume, for accessing CORS data. Users who download large quantities of CORS, often for research applications, find this mechanism to be faster and easier to automate into their own data management and access processes. In March, 2006, nearly 750 gigabytes of CORS-related data were downloaded via ftp.

In addition to providing access to GPS observation data, the CORS Web site also allows a user to view or download a variety of metadata and related information about each site. This information includes the following:

- data availability plots

- station coordinates and velocities of both the L1 phase center and the antenna reference point referenced to the North American Datum of 1983 (NAD83) and the International Terrestrial Reference Frame (ITRF)

- site logfile with equipment and installation details

- NGS station datasheet

- local and regional maps and site satellite imagery

- site photographs

- notices about site operation, outage forecasts, etc.

- 60-day station coordinate time series plot

- long-term station coordinate time series plot

- precise GPS satellite ephemeris data

The CORS Web site provides a wealth of CORS-specific and more general GPS-related information, in addition to sitespecific data and information. It includes a newsletter with updates about GPS and the CORS network; a list of frequently asked questions (and their answers); information for potential CORS collaborators; access to software; a list of related publications and presentations; links to other organizations and Web sites; a discussion of CORS coordinate issues; national and regional maps showing CORS site coverage; a list of forthcoming sites; and much more - including contact information for users to pose a question or provide a comment to CORS personnel.

A critical aspect of the CORS program is the computation, monitoring, and quality control of station positional coordinates and velocities. It is the combination of CORS
GPS observations and corresponding station coordinates that give the system its full value, for most users.

To satisfy the needs of the wide range of CORS users, NGS computes and provides station coordinates and velocities in two reference systems - ITRF and NAD83. ITRF was developed by the International Earth Rotation Service (IERS) and is widely utilized in the global research community, whereas NAD 83 was produced by NGS and is commonly used in surveying and mapping applications in the United States. A fundamental distinction between these two systems is that NAD83 is defined to be fixed with respect to the North American tectonic plate, while ITRF satisfies a global condition of zero net rotation of all of the plates. One result of this difference is the characteristic that, on most of the stable part of the North American plate (most of the United States, excluding portions or all of California, Oregon, Washington, Alaska, and Hawaii), NAD83 coordinates generally have zero, or negligible, velocities associated with them, and ITRF horizontal coordinates change at a rate of approximately 1-2 $\mathrm{cm}$ per year. Hence it is important to provide velocity estimates and specify an epoch date for coordinates.

CORS coordinates and velocities are provided for both ITRF and NAD83, in Earth-centered, Earth-fixed Cartesian coordinates $(\mathrm{X}, \mathrm{Y}, \mathrm{Z})$ and geodetic coordinates (latitude, longitude, and ellipsoid height - relative to the Geodetic Reference System of 1980 (GRS80) ellipsoid). Every few years IERS computes a global network solution that integrates measurements from a number of different geodetic technologies to generate a revised realization of ITRF. NGS is currently utilizing the 2000 realization (ITRF2000) referred to an epoch date of January 1, 1997, and will adopt future ITRF realizations, as they are released by IERS. The global precise positioning community is currently anticipating a near-term release of the 2005 realization of this system - the ITRF 2005 .

CORS coordinates are published for both the L1 phase center and the antenna reference point (ARP) of each site's GPS antenna. The ARP is usually the physical bottom of the antenna and is commonly used as the reference height level in GPS data processing. There are, however, some users and applications that prefer to reference the L1 electrical phase center of the antenna, so NGS provides both sets of coordinates.

Part of the process of incorporating a new station into the CORS network is to compute the station coordinates that will be published and provided to users. This initial determination of the station position is based on the processing of a minimum of about two weeks (and more in some areas, due to problematic atmospheric conditions, etc.) of GPS observations. The native result of this effort is a set of ITRF station coordinates. The velocities associated with the station are modeled using the NGS Horizontal Time-Dependent Positioning (HTDP) software. The NAD83 station position and velocities are then computed from the ITRF values using a Helmert transformation. Eventually, after a number of years, and the station has realized sufficient history in the CORS network, the ITRF and NAD83 modeled velocities are replaced by GPS-measured values. 
The accuracy and consistency of CORS site coordinates are continuously monitored by NGS - for both National and Cooperative CORS - through a daily solution of all stations and a rigorous least squares network adjustment. This adjustment is currently constrained to three well-determined CORS sites and two stations that are part of the cooperative network of the International Global Navigation Satellite System (GNSS) Service (IGS), thereby providing an important link between the CORS and a more global, research-focused GPS network. The recent behavior of each station's coordinates can be viewed in the 60-day time series plots, which show the differences between the daily ITRF solution, transformed in time back to the published position epoch date, and the published station coordinate values. The plotted values are the north, east, and up components, each characterized with an error bar and summarized by a statement of the 60-day mean (or bias) and RMS.

Approximately every year, NGS also computes a rigorous set of station coordinate values based on every third day of GPS observations over the history of every CORS. This allows the behavior of each site, over time, to be analyzed and the quality of its published position to be monitored. If the difference between a station's published coordinates and history of computed coordinates - based either on analysis of the 60-day plots or the multi-year solution - consistently exceeds $1 \mathrm{~cm}$ in either horizontal component or $2 \mathrm{~cm}$ in the vertical component, for ITRF, or $2 \mathrm{~cm}$ in either horizontal component or $4 \mathrm{~cm}$ in the vertical component, for NAD83, NGS will publish new coordinate values, and velocities, relative to the impacted reference frame(s). These coordinate republication criteria represent a compromise between maintaining coordinate consistency over time and providing the most accurate values possible. The coordinate behavior of each site, for its entire history, can be viewed in the long-term time series plots that are provided.

The ongoing monitoring and characterization of station behavior and the occasional republishing of CORS coordinates mean that users can be assured of a high degree of CORS coordinate quality. For this reason alone, a CORS is superior to a static ground control station, whose coordinates can become accuracy-degraded over time, relative to the requirements for some demanding applications. Even in areas that are prone to surface motions, due to tectonic, volcanic, fluid-withdrawal effects, etc., the CORS system provides station behavior characterization to support decisions about how best to utilize the station's coordinates. Some users of the CORS network exploit this coordinate behavior information through the computation of their own updated CORS coordinate values, based on statistics provided in the 60 -day plots. They, in effect, use the recent observed history of the site to fine-tune its coordinates for use in their project.

\section{Applications}

The primary motivation behind NGS' effort in building the CORS network has been to provide the infrastructure to allow the geospatial user community to improve the accuracy of GPS measurements through relative positioning techniques, based on simultaneous data collected at CORS sites. These user techniques can include long-session static positioning for the establishment of geodetic control (centimeter accuracy), kinematic travel-path determination of moving platforms (few-decimeter accuracy), and feature mapping for GIS data collection (meter to sub-meter accuracy). In each of these application categories, the power of the CORS network is realized through its ability to provide GPS observations, collected at precisely known locations, which substantially improve the accuracy of the user's GPS measurements.

Paralleling the ongoing growth of the CORS network is an expansion in the range of applications supported by CORS and CORS-derived data. The high accuracy of CORS coordinates and velocities coupled with the reliability and availability of the associated GPS observation data have enabled a broad array of applications, many of which were not envisioned during the early days of the CORS system - now a decade ago. With the ability to support centimeter-level accuracies, the system is now involved in disciplines far beyond the original focus of surveying- and mapping-related applications.

NOAA's Global Systems Division (http://www.fsl.noaa. gov/) conducts research and development to provide the nation with environmental information and forecast capabilities. The agency's Ground-Based GPS Meteorology program relies on CORS data, from hundreds of sites, to determine atmospheric water vapor content, derived from tropospheric GPS signal delays. This precipitable water data is now being used operationally to enhance meteorological forecast capability and has proven very valuable to the meteorological community, and hence to the nation.

NOAA's Space Environment Center (http://www.sec.noaa. gov/) is responsible for monitoring and forecasting Earth's space environmental conditions. CORS observations are now contributing prominently to this effort by providing the ability to characterize the total electron content (TEC) in the ionosphere. By exploiting the dispersive nature of the ionosphere and the delay effect it has on the GPS signals, ionospheric TEC can be calculated. GPS-derived TEC maps for the continental United States are now provided online and are updated on a 15-minute cycle with just 30-minute latency. This data is valuable to researchers, satellite operators, utility companies, and others who are impacted by ionospheric conditions.

Tropospheric and ionospheric modeling efforts are being further exploited to enhance GPS surveying capabilities. The ability to quantify atmospheric GPS signal delay can greatly benefit the GPS data processing task of resolving integer ambiguities, thereby allowing for a decrease in required occupation times. Additionally, for users of single frequency GPS equipment, CORS-derived atmospheric condition data can allow greater project distances to be covered.

CORS data are frequently used to provide GPS base station data to differentially correct GPS observations used in aircraft to locate airborne imagery. This application benefits greatly from the CORS network because of the simplification in project logistics that is realized by tapping into the existing CORS infrastructure. Aerial imagery providers can enjoy 
considerable time and cost savings by incorporating CORS data into their process and reducing or eliminating the requirement to establish their own project-specific base stations. Recovery work in the United States Gulf Coast region following the devastating 2005 hurricane season was bolstered by the collection of aerial imagery by the NGS Remote Sensing Division. This comprehensive set of aerial photographs, all of which were positionally controlled by regional CORS sites, was made available to the recovery effort with rapid turnaround - thanks, in part, to the CORS network.

NGS and many other organizations use observation data from the CORS network, and other permanent GPS networks, for the determination of precise GPS satellite orbits. These orbits, in turn, contribute to high accuracy positioning capability because the accuracy of GPS-derived coordinates on the ground is partially dependent on the accuracy of the satellite positions used in processing the observations. The IGS, an international federation of 200 organizations including NGS - involved in the production of a variety of GPS and GLONASS data products, coordinates the production of precise GPS satellite orbits which are derived from analysis performed by participating organizations located around the globe. The participation of the CORS network in a multinational, worldwide effort of developing GPS-related data products provides an important bridge between the United States-centric CORS network and the global space-based positioning arena.

GPS has become one of the tools of choice for the research community interested in measuring motions of the earth's surface, which are the result of tectonic deformation, postglacial rebound, groundwater withdrawal, or other natural or man-induced causes. The accuracy capabilities, in both the horizontal and vertical components, supported by the CORS network - and related continuous GPS networks - have made possible the monitoring of these motions and, in some cases, the determination of trends supporting some predictive capability. Many organizations and multi-organization cooperative endeavors are using permanent GPS networks to monitor motions. A few examples are: EarthScope/Plate Boundary Observatory (http://pboweb.unavco.org/), Pacific Northwest Geodetic Array (http://www.geodesy.cwu.edu/), Scripps Orbit and Permanent Array Center (http://sopac. ucsd.edu/), Bay Area Regional Deformation Network (http://www.ncedc.org/bard/), and Basin and Range Geodetic Network (http://cfa-www.harvard.edu/space_geodesy/ BARGN/). Many of the continuous GPS sites in these networks are also included in the CORS network, thereby furthering the cooperative, multi-use nature of these ambitious programs.

NGS is relying heavily on the CORS network in satisfying its federal requirement to provide the NSRS. CORS represents the highest accuracy component or tier of the NSRS. This importance of the CORS network is reflected in NGS' present effort to readjust the existing, GPS-observed ground control stations to produce a new realization of the NAD83 system. For this process, the published positions of all CORS sites will be held fixed and the monumented points, which have been observationally connected to the CORS system, will be adjusted to the CORS. The results of this readjustment will be an increase in the positional accuracy of the monumented points and a higher degree of consistency, than currently exists, between the coordinate values of the CORS and the monumented points.

\section{E. Future CORS Plans}

NGS is constantly trying to improve its products and services, including the CORS network. As previously mentioned, CORS personnel operationally monitor daily CORS solutions and long-term site behavior to track issues such as coordinate and velocity consistency and site stability. Reference [3] describes the ongoing effort of maintaining the most accurate coordinate set possible for the CORS network, and the authors emphasize that daily monitoring of coordinate variability is an essential part of providing a rigorous permanent network.

Reference [4] examines data from nearly 400 CORS sites to better understand signal multipath issues, and important conclusions were made regarding the impact of site issues such as monumentation, antenna configuration, and signal environment, and how they affect data quality. Ongoing research efforts, such as this, have contributed to the development of the aforementioned site guidelines document, which was developed to provide consistency and reliability throughout the CORS network. The guidelines will help to ensure that the system can support even the most accuracydemanding of applications well into the future.

Due in part to the ongoing interest in the development of local and regional permanent GPS networks, the CORS system will continue to grow and NGS will accommodate this growth to the extent possible. One near term goal of the CORS program is to improve the spatial coverage throughout the United States. Presently, some areas are considerably better served than others, because of the cooperative nature of the network. A level of station redundancy is desirable because, at any given time, a small percentage of stations might be non-operational due to site maintenance activity or equipment failure.

NGS also hopes to reduce the latency of data provided by stations. Although many stations provide data to NGS hourly, or in real time, there are still a number of sites that, due to communications limitations, are restricted to providing the data daily. As new sites are being considered for inclusion in the network, the data latency is one of the site issues that are examined.

In recent years, NGS has solicited feedback and recommendations from the CORS user-community, often at venues such as national conferences presented by organizations, including the Institute of Navigation and the American Congress on Surveying and Mapping. In order to determine how best to evolve the CORS system, NGS needs to know what the user community requires in reference station infrastructure and ideas garnered through these forums - and other means - have helped guide this process. 
One GPS issue that is of great interest, particularly in the surveying community, is the delivery of reference station data in real time, which is the foundation of the network real-timekinematic (RTK) approach to precise positioning. With the profusion of real-time networks, such as many state and regional entities are presently developing, there has been a profound paradigm shift in the precise positioning arena. And, NGS is exploring fundamental support, through the CORS network, to this technology.

Testing is currently underway to determine how NGS can provide real-time delivery of CORS GPS observation data via the Internet. One method that is being examined and considered is a Radio Technical Commission for Maritime Services (RTCM) standard protocol, known as Network Transport of RTCM via Internet Protocol (NTRIP). By utilizing a technique such as NTRIP, NGS could provide a real-time stream of CORS GPS observation data over the Internet that would be accessible to end users or to real-time service providers, who would process the data and deliver a derived product. It is important to note that, at this time, there are no plans for NGS to provide a real-time derived product, such as a network RTK solution, nor would the anticipated Internet data stream from NGS be suitable for applications such as navigation. NGS' involvement in the real-time arena is still very much open to discussion, evaluation, and consideration

IGS is interested in supporting a global, real-time positioning infrastructure and the CORS network could be an important component of such an undertaking. Such a lofty goal would require the participation of an unprecedented number of collaborators, but the result would indeed be profound.

Estimates of the tangible value of the benefits provided to the positioning community by the CORS network are now in the range of tens of millions of dollars per month. For taxpayers in the United States alone, this represents a huge return on the relatively minimal cost incurred by the program. As the network grows and more users find new ways to take advantage of CORS data, the direct value of the system will undoubtedly continue to increase.

\section{ONLINE POSITIONING USER SERVICE}

\section{A. Overview of the Utility}

The CORS program underwent a profound functional advancement in 2001 when NGS began offering enhanced access to the system through a powerful Web-based utility the Online Positioning User Service (OPUS). In developing OPUS, which is still substantially evolving, NGS' goal has been to provide the geospatial community with a simplified means of accurately accessing the NSRS through an automated process that provides consistent results with rapid turnaround.

With OPUS, users can submit their own GPS observations through a simple Web interface, requiring only minimal user input, and within minutes they will receive an email reporting the coordinates of their data collection site, computed via rigorous GPS vector connections to three CORS. This process is performed using NGS software and computers and is provided free of charge.

\section{B. User Interface}

The OPUS submittal Web page (www.ngs.noaa.gov/ OPUS/) can be link-accessed from several locations around the NGS Web site - principally the NGS homepage, the main CORS homepage, and the Geodetic Toolkit. This submittal page asks the user to enter the following basic information through a straightforward interface:

- email address to receive the results

- observation file(s) in RINEX or most receiver raw formats, optionally compressed

- vertical antenna height, in meters, from mark to ARP

- antenna type, chosen from dropdown menu

and the following information, which is optional:

- forced State Plane Coordinate System zone

- up to 3 specific CORS to include in the solution, which may include Cooperative CORS

- specific CORS to exclude from the solution

- whether extended output information is desired

- whether to capture user configuration in a profile

Once this information is compiled and uploaded to OPUS, the user simply waits for an email containing the results that will normally (depending on the current processing load) arrive within minutes.

Some guidelines/requirements governing user submissions are:

- static GPS observations

- dual-frequency (L1/L2) GPS observations

- minimum 2-hour span of data recommended

- epoch rate an integer divisor of 30 seconds

\section{The Process}

Final OPUS-derived coordinates reported back to the submitter are the average of separate single-baseline solutions connecting the user's GPS measurements to simultaneous measurements made at each of three CORS. These ionospheric-free solutions are generated by NGS' GPS processing software package, PAGES (Program for the Adjustment of GPS Ephemerides), utilizing doubledifferenced, carrier-phase measurements to compute ITRFreferenced baseline vectors.

Prior to processing the baselines, OPUS must first determine the CORS sites that PAGES will use. This is an iterative procedure involving a number of tests that examine the availability and quality of data collected at candidate stations, beginning with the optional user-specified sites, which may include Cooperative CORS, and the National CORS sites which are closest to the user's data collection location. Data quality issues that are investigated include signal multipath and cycle slips, the presence of which can degrade the results' accuracy. If the GPS data of the userselected CORS or the closest CORS are deemed insufficient in quality or quantity - the search is then expanded outward and other potential CORS data files are examined, until three sites with suitable data are identified. 
Typically, the result of the CORS selection process is that the three closest CORS are picked, but there are exceptions to this generalization. One such situation could occur if a user performs an OPUS submittal with very little delay following the end of the observing session. Because some CORS sites transmit data to NGS only daily, data from some nearby CORS might not yet be available for the required time period, and OPUS would then expand its search to more distant sites in order to find adequate data coverage.

It is important to note, however, that users are allowed to submit their data as soon as desired after it is collected. Because many CORS sites do provide data to NGS on an hourly or real-time basis, there will normally be a sufficient number of CORS with valid data to allow OPUS to work, even with very little time delay following the end of the user's session. The CORS utilized in such a situation, however, might be much more distant than they would be if the user were to wait a number of hours before submitting.

Although OPUS computes the inter-site vectors in the ITRF system, it produces final coordinate values referenced to both ITRF and NAD83. To derive the ITRF results, OPUS retrieves the published ITRF positions for the selected CORS from the NGS Integrated Database. These coordinates are then transformed to the epoch represented by the mid-point of the time span of the user's data file, to accommodate the motion-induced changes (e.g. approximately $1-2.5 \mathrm{~cm}$ annually on the North American tectonic plate) of ITRF coordinate values. The time-transformed coordinates are then used in the three distinct PAGES solutions that are ultimately averaged to determine the final ITRF coordinates.

Corresponding NAD83 coordinates (for the date of observation) are obtained by transforming the derived ITRF coordinates via the 14-parameter Helmert equations adopted for this purpose. These NAD83 coordinates are then projected to a standard epoch date of January 1, 2002, using the NAD83 velocity predicted by the HTDP software.

It is important to note that, for both ITRF and NAD83, the three distinct solutions, each based on a connection to one of the three CORS, are not completely independent of each other. This is due to the fact that all three solutions incorporate the same set of user-submitted observations and are all subject to the same potential biases at the user's data collection site. Possible biases include signal multipath caused by the site environment and an error in determining the horizontal and/or vertical relationship between the user's GPS antenna and the ground monument, resulting, respectively, in centering or antenna height errors. The ultimate effect of such biases would not be averaged out or mitigated through a combination of the three distinct solutions and would likely go undetected.

\section{Output}

The OPUS output page contains a concise, yet thorough, summary of the results of the automated processing of the submitted observation file. In addition to echoing back all of the user's input information, the output includes the following:

- GPS satellite ephemeris used

- start/end time of observation file
- number of observations used/present and $\%$ used

- number of ambiguities fixed/total and \% fixed

- overall RMS of vector processing

- NAD83 and ITRF coordinates in (X, Y, Z) and latitude, longitude, ellipsoid height

- NAVD orthometric height based on Geoid03 model

- peak-to-peak error for all coordinate components

- UTM and SPCS metric planar coordinates, convergence, and scale factors

- U.S. National Grid designator

- name, position, and distance for each of the 3 CORS

- nearest published NGS control point.

And if the user requests the optional extended output, the following information is included in the email:

- for each CORS, summary of derivation of timetransformed ITRF coordinates

- for user's location, summary of derivation of timetransformed starting coordinates

- for each baseline, summary of derivation of user's coordinates

- G-file vector records for use in NGS Adjust software

- Post-fit RMS satellite vs. baseline results

- summary of number of observations for each satellite vs. baseline

- covariance matrix elements for inclusion in network adjustment software

- estimate of horizontal and vertical network accuracies

- summary of derivation of NAD83 vector components

- SPCS planar coordinates in feet, if a specific statespecified foot conversion exists

\section{E. Results}

The quality of the computed coordinate components is characterized in the OPUS output by comparing the results of the three individual solutions and computing the peak-to-peak errors of the $\mathrm{X}, \mathrm{Y}$, and $\mathrm{Z}$ geocentric values and the latitude, longitude, ellipsoid height, and orthometric height (NAD83 solution only) values. Peak-to-peak errors are simply the difference between the maximum and minimum value for each coordinate component.

The peak-to-peak error statements included in the output provide a more meaningful characterization of the quality of results compared with the formal errors derived from GPS vector processing, which are known to be unrealistically optimistic. In addition to providing more realistic estimates of the precision of the solutions, the peak-to-peak errors have the added advantage that they also reflect any error in the CORS coordinate values used in the solutions.

The achievement of quality OPUS results is, in part, dependent on freedom from any systematic error in the process. Most typical user-caused potential errors are related to the GPS antenna - errors in measuring or inputting the mark-to-antenna (measured at the ARP, in meters) vertical offset, centering the antenna over the mark, or selecting the antenna type on the OPUS submittal page. The use of an incorrect antenna type alone, which would cause OPUS (and PAGES) to use the wrong antenna electrical phase center 
model during processing, could lead to an error in height as great as $10 \mathrm{~cm}$, or more. Systematic errors such as these would neither be detected by OPUS nor would they be reflected in the peak-to-peak error statements.

The following list provides a guideline set of minimum OPUS output criteria that will help the user identify when an OPUS run has been successful:

- $>90 \%$ of submitted observations used

- $>50 \%$ of integer ambiguities fixed

- $<3 \mathrm{~cm}$ overall RMS

- $<5 \mathrm{~cm}$ peak-to-peak errors, in each component

- confirm correct antenna type and height were input.

For OPUS users desiring to better their results, the routine advice to be given is quite simple: collect more data. Reference [5] presents an investigation to determine the relationship between the duration of observing session and coordinate accuracy achieved by OPUS. By utilizing 30 days of CORS data, subdivided into sessions of $1,2,3$, and 4 hours and submitting these test datasets to OPUS, the authors compared the results with the published positions of the CORS sites, which were the sources of the test data. For sessions of 2 hours, the RMS results of $0.8 \mathrm{~cm}, 2.1 \mathrm{~cm}$ and $3.4 \mathrm{~cm}$ in the north, east, and vertical components, respectively, corroborated the OPUS guidelines suggesting 2 hours as the minimum time needed to typically provide sufficient accuracy for many surveying-related tasks. Applications that are more demanding, in terms of accuracy, will require longer datasets. The results for the 3- and 4-hour sessions show substantial improvement compared with the 2-hour sessions. Furthermore, the study shows that a reduction of session length to 1 hour greatly deteriorates the accuracy, due primarily to the inability of the software to adequately determine the integer ambiguities.

Another NGS study, by OPUS personnel, involved the submission to OPUS of over 2002 -hour test datasets, again collected at CORS sites, to examine the accuracy achieved with the recommended session length. The results - RMS values of $0.8 \mathrm{~cm}, 1.4 \mathrm{~cm}$, and $1.9 \mathrm{~cm}$ in the north, east, and vertical components - were slightly better, particularly in the vertical component, than the results of [5].

Other studies, such as [6], have shown that for static GPS processing, there is little relationship between coordinate accuracy achieved and GPS vector length, assuming that there is adequate observation data to fix the integer ambiguities and to correctly estimate the tropospheric delay.

The impact of the results of these aforementioned studies is that OPUS users can expect to achieve good results as long as they have collected and submitted a sufficient (sufficiency being determined by the user's specific application of the results) quantity of data from a quality collection site, even if OPUS uses CORS sites in the solution that are a great distance from the user's site.

In addition to session length, another issue that can impact OPUS results is the GPS satellite orbits that are used in the processing. The orbits come in three different varieties, all available from IGS, and they are listed here in increasing order of accuracy, along with their typical availability delays:
- IGS ultra-rapid orbits (no delay)

- IGS rapid orbits (1 day)

- IGS precise orbits (10-14 days)

OPUS will always use the best available orbit file for processing. If an OPUS submission is made within a few hours after the end of the user's observing session, for example, OPUS will use the ultra-rapid orbit file because that is the only version that is available. If, however, there is a delay of a few days before the submission, the rapid orbits will then be available and will be used. The OPUS output will report which orbit file was used.

For most users, the resulting coordinate accuracy improvement realized in going from a solution based on the ultra-rapid orbits, which are partially predicted, to the rapid orbits, which are based fully on observations, warrants the effort of waiting a day or so and resubmitting the file to OPUS for reprocessing with the improved orbits. The effective improvement for most users (working with baselines up to several hundred kilometers in length), however, in going from a solution based on the rapid orbits to one utilizing the final precise orbits is so minimal that resubmission is typically not warranted.

OPUS processing utilizes 30 -second epoch rates and, although users often collect data at a faster rate, there is no advantage (nor is any harm done, assuming the epoch rate in the submitted file is an integer divisor of 30) to submitting higher-rate data.

\section{F. Future OPUS Plans}

The popularity of OPUS has been increasing substantially with time and usage is now typically in the range of 10,000 to 15,000 files processed per month, with a range of 2,000 to 3,000 users per month. Fig. 3 shows the history of the number of files processed by OPUS per month for the past 4 years. Usage has become more widespread, both geographically and in the variety of applications for which it is being employed. This growth is at least partially a result of the expansion of the CORS network, but is also due to the fact that as more people

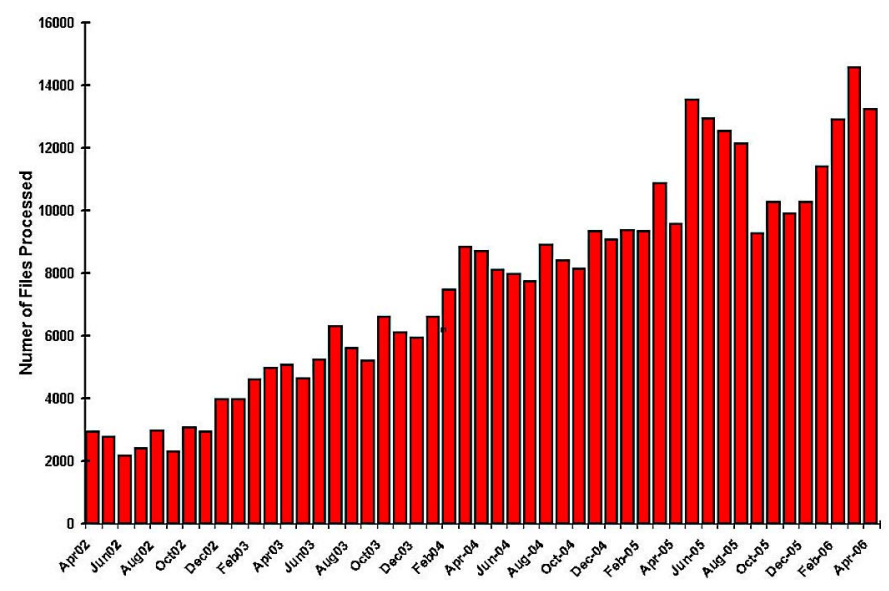

Fig. 3. Chart of number of monthly OPUS files processed for April, 2002 through April, 2006. 
learn about the utility and how it can be used, they are putting it to work. Reference [7] is a good overview of the utility that provides exposure to the general surveying community, the largest single group of OPUS users. Additionally, [8] presents the utility to an audience that is more likely to be focused on defense and intelligence applications of positioning technologies. As an example of the recognized importance of OPUS, it was selected as the March, 2004, "Internet Resource of the Month" by GEO World magazine.

Most OPUS users employ the utility simply to statically establish their own geodetic control for various surveyingrelated project applications. OPUS is extremely powerful in its support of this kind of work. Some users are going a step further in how they use OPUS by doing things such as integrating the utility into their own RTK GPS work. In this approach, surveyors can set up an RTK base station anywhere, perform the RTK survey based on an approximate, autonomous position for the base station, and after returning to the office, submit the base station data to OPUS to determine the base coordinates. The RTK rover data can then be reprocessed utilizing the new, OPUS-derived base station coordinates to correct the rover data for the entire project.

NGS presently has tentative plans for several future versions of OPUS that will greatly improve its usefulness and flexibility. In its present configuration, OPUS requires the use of dual-frequency, survey-grade GPS receivers, at least two hours of GPS observations, and it delivers the results to the submitter only and not to any database. In the proposed forthcoming enhanced OPUS versions, each of these characteristics will be modified. It is important to note that the following proposed OPUS versions, their capabilities, and names are all subject to change. Implementation of any of these changes to OPUS will depend on NGS policies which have not yet been established.

With "OPUS-Database," qualified submitters will be able to register in order to have their OPUS results captured into the NGS database for others to access. Database entries will be restricted to OPUS submission results that meet specific quality criteria. The "OPUS-Database" results can complement geodetic control data pertaining to stations already existing in the NGS database or they can be used to establish new points not previously contained in the database. An example of how "OPUS-Database" might be used to improve data on an existing point would the OPUSpositioning of a vertical control point, whose existing horizontal position of record was derived by scaling from a topographic map. By capturing the OPUS results into the database, the recorded horizontal position of the point would be upgraded from the approximate, scaled coordinates to the more accurate OPUS values. Furthermore, the OPUS-derived ellipsoid height would also be captured, thereby providing an observed determination of the station's geoid height (the difference between the existing orthometric height and the newly established ellipsoid height) - an important contributor to future geoid models. "OPUS-Database" represents a vastly simplified procedure for capturing geodetic control into the
NGS database, compared with the existing process that is required of all submitted projects.

"OPUS-Rapid Static" is being designed to provide precise positioning capabilities with occupation times around 15 minutes, instead of the currently recommended 2 hours. This capability will come from the use of a different processing engine, instead of PAGES, that will utilize the P1 and P2 pseudorange measurements in addition to the L1 and L2 carrier phase observations.

"OPUS-Projects" will allow users to submit simultaneous observations collected by their multiple receivers and the utility will process the connections between these multiple receivers as well as the connections to the CORS sites. A user's multiple observation sessions can be accumulated, thereby allowing for the establishment of a geodetic network approach to establishing control stations, including rigorous network adjustment analysis.

"OPUS-GIS" will be of most interest to the mapping and GIS communities. It will involve the use of short, approximately 1 minute, occupation times with code data from mapping-grade GPS equipment to achieve anticipated positional accuracy of a meter or better.

Each of these potential new varieties of OPUS will have specific applications for which it is best suited. The commonality for all of them is that they provide fast, easy, and reliable access to the NSRS and ultimately accurate geographic positions.

\section{CONCLUSIONS}

GPS technology has revolutionized the positioning and navigation arenas. The CORS network provides the infrastructure that supports even the most accuracy-demanding of location-based applications. Furthermore, OPUS gives users a fast and easy way to access the NSRS, via the CORS network. As the CORS network continues to evolve, and as applications of the CORS system expand, NGS will endeavor to provide enhanced user access to a consistent and reliable national positioning framework for years to come.

\section{ACKNOWLEDGMENT}

I thank NGS employees Cindy Craig, Ruth Osborne, Dr. Dru Smith, Dr. Richard Snay, and Neil Weston for their assistance. I appreciatively recognize Bill Strange, retired NGS chief geodesist, for long ago having the insightful vision of a nationwide CORS network, which has indeed come to pass.

\section{REFERENCES}

[1] R.A. Snay, G. Adams, M. Chin, S. Frakes, T. Soler, and N.D. Weston, "The synergistic CORS program continues to evolve," Proc., ION GPS 2002 (CD-ROM), Institute of Navigation, Alexandria, VA, pp. 26302639, 2002.

[2] "Guidelines for new and existing Continuously Operating Reference Stations (CORS)," National Geodetic Survey, 33 pp., February, 2006 (http://www.ngs.noaa.gov/CORS/Establish_Operate_CORS.html). 
[3] T. Soler, R.A. Snay, R.H. Foote, and M.W. Cline, "Maintaining accurate coordinates for the National CORS network," Proc. FIG Working Week, Paris, France, 8 pp., April 13-17, 2003.

[4] S. Hilla and M. Cline, "Evaluating pseudorange multipath effects at stations in the National CORS Network," GPS Solutions, 7(4), pp. 253$267,2004$.

[5] T. Soler, P. Michalak, N.D. Weston, R.A. Snay, and R.H. Foote, "Accuracy of OPUS solutions for 1- to 4-h observing sessions," GPS Solutions, 10(1), pp. 45-55, 2006.

[6] M.C. Eckl, R. Snay, T. Soler, M.W. Cline, and G.L. Mader, "Accuracy of GPS-derived relative positions as a function of interstation distance and observing-session duration," Journal of Geodesy, 75(12), pp. 633640, 2001.

[7] G.L. Mader, N.D. Weston, M.L. Morrison, and D.G. Milbert, "The Online Positioning User Service (OPUS)," Professional Surveyor Magazine, pp. 26-30, May, 2003.

[8] G.L. Mader and N.D. Weston, "NGS' Online Positioning User Service," GeoIntelligence, pp. 16-19, January-February, 2006. 